\title{
Sarcoidosis - A Case Report
}

\author{
*MS Islam ${ }^{1}$, MMR Siddiqui ${ }^{2}$
}

\begin{abstract}
Sarcoidosis is a chronic granulomatous disorder of unknown etiology, commonly affecting the lungs, skin and eyes. Although lungs and lymph nodes are involved in more than $90 \%$ of patients, virtually any organ can be involved. We describe a 36 years old lady presented with fever, skin rashes, cough, polyarthralgia, bodyache, wt. loss for 3 months. Examination revealed Fever, Erythema Nodosum, Cervical Lymphadenopathy. Investigations revealed high ESR (80 mm in 1st hour), high CRP, Hilar Lymphadenopathy on Chest X ray, Non-caseous Granuloma, Giant cell and Asteroid body on Lymph node Biopsy. All of her history, clinical examinations and Investigations are suggestive of Sarcoidosis. With symptomatic treatment and watchful observation, now she is completely symptoms free and leaving a healthy life.
\end{abstract}

Key Words: Fever, Erythema Nodosum, polyarthralgia, Sarcoidosis

\section{Introduction}

Sarcoidosis is a multisystem disease that leads to inflammation, usually in the lungs, skin, or lymph nodes. It starts as tiny, grain-like lumps, called granulomas. ${ }^{1}$ Sarcoidosis can affect any organ in body. No one is sure what causes sarcoidosis. It affects men and women of all ages and races. ${ }^{2,3}$ It occurs mostly in people ages 20 to 50 years, African Americans and people of Northern European origin. Many people have no symptoms. If you have symptoms, they may include Cough, Shortness of breath, Weight loss, Night sweats, Fatigue. Tests to diagnose sarcoidosis include chest $\mathrm{x}$-rays, lung function tests, and a biopsy. Not everyone who has the disease needs treatment. However, prednisone, a type of steroid, is the main drug of choice. We describe a 36 years old lady presented with fever, skin rashes, cough, polyarthralgia, bodyache and wt. loss for 3 months. All of her history, clinical examinations and Investigations are suggestive of Sarcoidosis. With symptomatic treatment and watchful observation, now she is completely symptoms free and leaving a healthy life.

\section{Case Report}

36 years of age normotensive, nondiabetic women came to department of Medicine, Bikrampur Bhuiyan Medical College Hospital with the complaints of fever, cough, multiple joint pain, skin rashes over both legs, wt. loss for last 3 months. Fever was low grade continuous in nature. Cough was non-productive and no diurnal variation. Painful tender nodular reddish skin rashes appeared specially over shins. There was pain in multiple joints involving wrist, elbow and ankles with early morning stiffness but no joint swelling observed. She got significant wt. loss, $3 \mathrm{~kg}$ in 3 months. No history of eye problem, no cardiac or neurological problem. Bladder and bowel functions were normal. Examination revealed average built, oriented young lady with tender nodular reddish rashes over both shins. The patient was febrile $100^{\circ} \mathrm{F}$ with regular heart rate $90 \mathrm{~b} / \mathrm{min}$, blood pressure of $110 / 70 \mathrm{mmHg}$ and normal jugular venous pressure, but there were cervical lymphadenopathy appeared 2 months later of initial symptoms involving bilateral anterior,

$1 * D r$. Md. Safiqul Islam, Assistant Professor, Department of Medicine, Bikrampur Bhuiyan Medical College and Hospital

${ }^{2}$ Dr. Md. Mahmudur Rahman Siddiqui, Associate Professor of Medicine, Anwer Khan Modern Medical College and Hospital

*Corresponding Author

Date of submission: 04.09.2018 Date of acceptance: 16.11.2018

AKMMC J 2019; 10(1) : 92-95 
posterior cervical groups. Joint pain present on movement of ankle and wrist but no joint swelling, there was full range of movements of all locomotor system and no proximal or distal muscular weakness. Examination of chest, abdomen, central and peripheral nervous systems were unremarkable. Investigations revealed haemoglobin $11.5 \mathrm{gm} / \mathrm{dl}$, erythrocyte sedimentation rate (ESR) $80 \mathrm{~mm}$ in 1st hour, total white blood cell count $7.71 \times 10^{9} / \mathrm{L}$, neutrophil 58\%, lymphocyte 29\%, CRP high but Tuberculin skin test or Mantoux Test was negative $(0 \mathrm{~mm})$. Initial Chest $\mathrm{X}$ ray on $06-11-17$ was normal but later on 17-12-17 revealed Bilateral Hilar Lymphadenopathy. Urine routine examination was normal, no growth in urine culture, Serum Calcium and 24 hours urinary total calcium was normal. Liver function test was normal, RA test - negative, ANA - negative, ultrasonography of the whole abdomen was normal. Finally Lymph Node Biopsy done- non-caseating granuloma, Giant cell, Asteroid body were found.

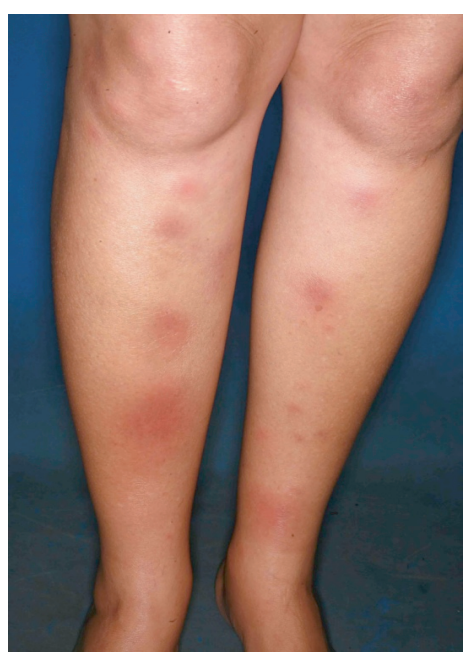

Fig: Patient of Sarcoidosis with Erythema Nodosum.

Finally according to sign, symptoms and investigations -Sarcoidosis diagnosis was made. We have started oral prednisolone in lowest possible dose for six months and she responded significantly. In the meantime she became afebrile, cough improved a lot, Rash and lymph nodes disappeared. CBC repeated - ESR $40 \mathrm{~mm}$ in $1^{\text {st }}$ hour, CRPnormal 0.49 (0.01-0.5 normal range). Now she is completely symptoms free and leaving a healthy life. She was advised for regular follow-up.

\section{Discussion}

Sarcoidosis is a multisystem granulomatous disorder of unknown aetiology characterised by the presence of non-caseating granulomas. Granulomatous inflammation is characterized primarily by accumulation of monocytes, macrophages, and activated T-lymphocytes, with increased production of key inflammatory mediators, TNF, IFN- $\gamma$, IL-2, IL-8, IL-10, IL-12, IL-18, IL-23 and TGF- $\beta$, indicative of a Th1-mediated immune response. The disease is more common in African Americans and white people of Scandinavian heritage. More women than men have the disease. The disease often begins between ages 20 and 40. Sarcoidosis is rare in young children. The disease usually begins in the lungs, skin, or lymph nodes. Less commonly affected are the eyes, liver, heart, and brain. Any organ, however, can be affected. There may be no symptoms. When symptoms do occur, they can involve almost any body part or organ system. The signs and symptoms depend on the organ involved. Almost all people affected by sarcoidosis have lung or chest symptoms. Common symptoms, which tend to be vague, include fatigue (unrelieved by sleep; occurs in $66 \%$ of cases), lack of energy, weight loss, joint aches and pains (which occur in about $70 \%$ of cases), ${ }^{4}$ arthritis (14-38\% of persons), dry eyes, swelling of the knees, blurry vision, shortness of breath, a dry, hacking cough, headache, Seizures, Weakness on one side of the face or skin lesions. ${ }^{5,6,7,8}$ Less commonly, people may cough up blood. ${ }^{5}$ The cutaneous symptoms vary, and range from rashes and nodule (small bumps) to erythema nodosum, granuloma annulare, or lupus pernio. The combination of erythema nodosum, bilateral hilar lymphadenopathy, and joint pain is called Löfgren syndrome, which has a relatively good prognosis. ${ }^{5}$

The diagnosis of sarcoidosis can occasionally be made on clinical grounds without a confirmatory biopsy when very specific clinical findings are present. Otherwise the diagnosis requires histological evidence of granulomatous inflammation, exclusion of alternative causes, and evidence of systemic disease. ${ }^{9}$ Isolated involvement of a single organ or organ system is rare in 
sarcoidosis. Therefore, all patients must be thoroughly assessed for additional disease manifestations. Lymphopenia is characteristic and liver function tests may be mildly deranged. Hypercalcaemia may be present. Hypercalciuria may also be seen and may lead to nephrocalcinosis. Serum ACE may provide a nonspecific marker of disease activity and can assist in monitoring the clinical course. Chest radiography has been used to ${ }^{10}$ stage sarcoid:

- Stage 0 - normal findings on chest radiograph.

- Stage I - bilateral hilar lymphadenopathy (which may be accompanied by paratracheal adenopathy).

- Stage II - bilateral hilar adenopathy with pulmonary infiltrates (parenchymal involvement or reticular opacities).

- Stage III - parenchymal infiltrates without hilar adenopathy.

- Stage IV - parenchymal involvement turns into volume loss (pulmonary fibrosis) and there may be other features (cavitations, calcifications, hilar retraction, bullae, cysts and emphysema).

In patients with pulmonary infiltrates, pulmonary function testing may show a restrictive defect accompanied by impaired gas exchange. Exercise tests may reveal oxygen desaturation. Bronchoscopy may demonstrate a 'cobblestone' appearance of the mucosa, and bronchial and transbronchial biopsy usually shows non-caseating granulomas. The BAL fluid typically contains an increased CD4: CD8 Tcell ratio. Characteristic HRCT appearances include reticulonodular opacities that follow a perilymphatic distribution, centred on bronchovascular bundles and the subpleural areas. The occurrence of erythema nodosum with BHL on chest X-ray is often sufficient for a confident diagnosis, without recourse to a tissue biopsy. Similarly, a typical presentation with classical HRCT features may also be accepted. Otherwise, the diagnosis should be confirmed by histological examination of the involved organ. The presence of anergy (e.g. to tuberculin skin tests) may support the diagnosis.

Sarcoidosis symptoms will often get better without treatment. Patients who present with acute illness and erythema nodosum are treated with NSAIDs and, if disease is severe, a short course of corticosteroids. Prednisolone (at a starting dose of 20-40 mg/day) should be commenced immediately in the presence of hypercalcaemia, pulmonary impairment, renal impairment and uveitis. This medicine may need to be taken for 1 to 2 years. ${ }^{11,12}$ The antimetabolites (eg, methotrexate, azathioprine, leflunomide and mycophenolate) are often used as alternatives to steroids. For patients who cannot be treated with low-dose glucocorticoids and an antimetabolite, anti-tumour necrosis factor (TNF) monoclonal antibodies have been shown to control disease. The next step in treatment includes new strategies such as rituximab. In rare cases, people with very severe heart or lung damage (end-stage disease) may need an organ transplant.

\section{Conclusion}

The disease can remit spontaneously or become chronic, with exacerbations and remissions. In some persons, it can progress to pulmonary fibrosis and death. In benign cases, remission can occur in 24 to 36 months without treatment but regular follow ups are required. Some cases, however, may persist several decades. ${ }^{4}$ Two-thirds of people with the condition achieve a remission within 10 years of the diagnosis. ${ }^{13}$ When the heart is involved, the prognosis is generally less favourable, though corticosteroids appear effective in improving $\mathrm{AV}$ conduction. ${ }^{14,15}$ The prognosis tends to be less favourable in African Americans than in white Americans. Our case is prognostically better but should be on regular follow up.

\section{References}

1. Valeyre D, Prasse A, Nunes H, et al. Sarcoidosis. Lancet. 2013 Sep 30. pii: S0140-6736(13) 60680-7. doi: 10.1016/S0140-6736(13) 60680-7.

2. Judson MA, Morgenthau AS, Baughman RP. Sarcoidosis. In: Broaddus VC, Mason RJ, Ernst JD, et al., eds. Murray and Nadel's Textbook of Respiratory Medicine. $6^{\text {th }}$ ed. Philadelphia, PA: Elsevier Saunders; 2016, chap 66.

3. Soto-Gomez N, Peters JI, Nambiar AM. Diagnosis and management of sarcoidosis. Am Fam Physician. 2016; 93(10): 840-848. PMID: 27175719 www. ncbi.nlm.nih.gov/ pubmed/ 27175719. 
4. Nunes H, Bouvry $\mathrm{D}$, Soler $\mathrm{P}$, et al. "Sarcoidosis". Orphanet Journal of Rare Diseases. Nov 2007; 2: 46. doi:10.1186/17501172-2-46. PMC 2169207. PMID 18021432.

5. King, TE Jr. (March 2008). "Sarcoidosis: Interstitial Lung Diseases: Merck Manual Home Edition". The Merck Manual Home Edition. Merck Sharp \& Dohme Corp. Archived from the original on 20 February 2014. Retrieved 19 February 2014.

6. Sweiss NJ, Patterson K, Sawaqed R, et al. "Rheumatologic manifestations of sarcoidosis". Seminars in Respiratory and Critical Care Medicine. August 2010; 31(4): 463-73. doi: 10.1055/s-0030-1262214. PMC 3314339. PMID 20665396.

7. Holmes J, Lazarus A. "Sarcoidosis: extrathoracic manifestations". Disease-A-Month. Nov 2009; 55(11): 675-92. doi:10.1016/j.disamonth. 2009.05.002. PMID 19857642.

8. Dempsey OJ, Paterson EW, Kerr KM, et al. "Sarcoidosis". BMJ. Aug 2009; 339: b3206. doi: 10.1136/bmj.b3206. PMID 19717499.

9. Judson MA. "Corticosteroids in Sarcoidosis". Rheumatic Diseases Clinics of North America. Feb 2016; 42(1): 119-35, ix. doi: 10.1016/j.rdc.2015.08.012. PMID 26611555.
10. Rao DA, Dellaripa PF. Extrapulmonary manifestations of sarcoidosis. Rheum Dis Clin North Am. 2013 May 39(2): 277-97. doi: 10.1016/j.rdc.2013.02.007. Epub 2013 Mar 13.

11. Baughman RP, Grutters JC. New treatment strategies for pulmonary sarcoidosis: antimetabolites, biological drugs, and other treatment approaches. Lancet Respir Med. Oct 2015; (10): 813-22. doi: 10.1016/S22132600(15) 00199-X. Epub 2015 Jul 20.

12. Beegle SH, Barba K, Gobunsuy $\mathrm{R}$, et al. Current and emerging pharmacological treatments for sarcoidosis: a review. Drug Des Devel Ther. Apr 2013; 127: 325-38. doi: 10.2147/DDDT. S31064. Print 2013.

13. "What Is Sarcoidosis?". National Heart, Lung and Blood Institute. National Institutes of Health. 14 June 2013. Archived from the original on 27 February 2014. Retrieved 21 February 2014.

14. Syed J, Myers R. "Sarcoid heart disease". The Canadian Journal of Cardiology. Jan 2004; 20(1): 89-93. PMID 14968147.

15. Sadek MM, Yung D, Birnie DH, et al. "Corticosteroid therapy for cardiac sarcoidosis: a systematic review". The Canadian Journal of Cardiology. Jan 2013; 29(9): 1034-41. 